\title{
A NOTE ON THE HOMEOMORPHISM GROUP OF THE RATIONAL NUMBERS
}

WAYNE R. PARK

\begin{abstract}
Let $Q$ be the rational numbers with the usual topology, $H(Q)$ the group of homeomorphisms of $Q, \gamma_{c}$ the convergence structure of continuous convergence, and $\sigma$ the coarsest admissible convergence structure which makes $H(Q)$ a convergence group. A counterexample is constructed to show that if $\kappa$ is a convergence structure on $H(Q)$ such that $\gamma_{c} \leqq \kappa \leqq \sigma$, then $\kappa$ is never principal, hence never topological.
\end{abstract}

In a previous work by the same author [4], a convergence structure $\sigma$ on $H(X)$, the group of homeomorphisms of the convergence space $X$, was developed which is the coarsest of the admissible convergence group structures on $H(X)$. When $X$ is a locally compact topological space, the $\sigma$ convergence structure becomes a topology, specifically the $g$-topology [1]. What happens to $\sigma$ when $X$ is no longer locally compact was left as an open question. Here a simple counterexample describes how badly nontopological the situation is when $X=Q$, the rational numbers with the usual topology. We use the notation as given in [3] and [4].

Let $H(X)$ represent the group of homeomorphisms of the convergence space $(X, \tau)$.

Definition. For each $f \in H(X)$ let $\gamma_{c} f$ consist of all filters $\mathscr{F}$ on $H(X)$ such that

(1) for all $x \in X$ and for all $\Phi \in \tau x, \mathscr{F}(\Phi) \in \tau f(x)$.

Here $\mathscr{F}(\Phi)=\omega(\mathscr{F} \times \Phi)$ where $\omega: H(X) \times X \rightarrow X$ is the evaluation mapping. The convergence structure $\gamma_{c}$ is called the convergence structure of continuous convergence [2].

Definition. For each $f \in H(X)$ let $\sigma f$ consist of all filters $\mathscr{F}$ on $H(X)$ such that $\mathscr{F}$ and $\mathscr{F}^{-1}$ simultaneously belong to $\gamma_{c} f$.

Here $\mathscr{F}^{-1}$ is the filter with filter base $\left\{F^{-1} \mid F \in \mathscr{F}\right\}$ where $F^{-1}=$ $\left\{f^{-1} \in H(X) \mid f \in F\right\}$.

It is clear that $\sigma$ is a finer convergence structure than $\gamma_{c}\left(\gamma_{c} \leqq \sigma\right)$. Moreover for $X=Q$, the rational numbers with the usual topology, $\sigma$ is strictly finer than $\gamma_{c}$ [4]. It is known that $\gamma_{c}$ on $H(Q)$ is not a topology [1], and

\footnotetext{
Presented to the Society, January 19, 1972 under the title Convergence structures on homeomorphism groups; received by the editors June 5, 1972 and, in revised form, January 2, 1973.

AMS (MOS) subject classifications (1970). Primary 54A20, 57E05.
}

(c) American Mathematical Society 1974 
moreover is not even a principal convergence structure [2]. Here we show the same is true of $\sigma$ on $H(Q)$, and in fact, true for any convergence structure between $\gamma_{c}$ and $\sigma$.

THEOREM. If $\kappa$ is a convergence structure on $H(Q)$ such that $\gamma_{c} \leqq \kappa \leqq \sigma$, then $\kappa$ is not a principal convergence structure.

Proof. Let $I$ be the set of irrational numbers. For each $\alpha \in I$, define a sequence of homeomorphisms $f_{n, \alpha}$ in $H(Q)$ by

$$
\begin{aligned}
f_{n, \alpha}(x) & =-x & & \text { if }|\alpha|-1 / n<|x|<|\alpha|+1 / n, \\
& =x & & \text { otherwise, }
\end{aligned}
$$

where $x \in Q$, and the integer $n$ is larger than $1 /|\alpha|$.

For each $\alpha \in I$, let $F_{N, \alpha}=\left\{f_{n, \alpha} \in H(Q) \mid n \geqq N\right\}$ and let $\mathscr{F}_{\alpha}$ be the filter generated by the filter base $\left\{F_{N, \alpha}\right\}$ where $N$ runs through the integers greater than $1 /|\alpha|$. As $f_{n, \alpha}^{-1}=f_{n, \alpha}$, it follows that $\mathscr{F}_{\alpha}=\mathscr{F}_{\alpha}^{-1}$ for each $\alpha \in I$. Moreover, $\mathscr{F}_{\alpha} \in \gamma_{c}$ (id) where id denotes the identity homeomorphism. Namely, let $q \in Q$ and let $\Phi$ denote the neighborhood filter of $q$ with the usual topology (convergence structure) $\tau$. To satisfy condition (1) above, let $U$ be arbitrary in $\Phi$. One can find $N$ sufficiently large and a $V$ in $\Phi$ such that $\omega\left(F_{N, \alpha} \times V\right) \subseteq U$. Hence $\mathscr{F}_{\alpha}(\Phi) \geqq \Phi$, so $\mathscr{F}_{\alpha}(\Phi)$ converges topologically to $\operatorname{id}(q)=q$, that is, $\mathscr{F}_{\alpha}(\Phi) \in \tau \operatorname{id}(q)$.

Now as $\mathscr{F}_{\alpha}=\mathscr{F}_{\alpha}^{-1}$ and as $\mathscr{F}_{\alpha} \in \gamma_{c}\left(\right.$ id), it follows that $\mathscr{F}_{\alpha} \in \sigma($ id) for each $\alpha \in I$.

But now, if $q$ is any nonzero rational number and $\Phi$ its neighborhood filter, $\omega\left(\bigwedge_{\alpha} \mathscr{F}_{\alpha} \times \Phi\right)=\left(\bigwedge_{\alpha} \mathscr{F}_{\alpha}\right)(\Phi)$ does not converge to id $(q)=q$. If it did, for any $U$ in $\Phi$ we would need to find a $V$ in $\Phi$ and a filter base element $\bigcup_{\alpha} F_{N_{\alpha}, \alpha}$ in $\bigwedge_{\alpha} \mathscr{F}_{\alpha}$ such that $\omega\left(\bigcup_{\alpha} F_{N_{\alpha}, \alpha} \times V\right) \subseteq U$. As irrationals lie arbitrarily close to $q$, this step is impossible.

We have constructed a family of filters $\left\{\mathscr{F}_{\alpha}\right\}$ such that $\mathscr{F}_{\alpha} \in \sigma(\mathrm{id})$ for each $\alpha \in I$ but such that $\bigwedge_{\alpha} \mathscr{F}_{\alpha} \notin \gamma_{c}$ (id). This shows that $\kappa$ cannot be a principal convergence structure.

\section{BIBLIOGRAPHY}

1. R. F. Arens, Topologies for homeomorphism groups, Amer. J. Math. 68 (1946), 593-610. MR 8, 479.

2. C. H. Cook and H. R. Fischer, On equicontinuity and continuous convergence, Math. Ann. 159 (1965), 94-104. MR 31 \#3995.

3. H. R. Fischer, Limesräume, Math. Ann. 137 (1959), 269-303. MR 22 \#225.

4. W. R. Park, Convergence structures on homeomorphism groups, Math. Ann. 199 (1972), 45-54.

Department of Mathematics, St. Lawrence University, Canton, New York 13617 